\title{
Virtual reality exercise on a home-based phase III cardiac rehabilitation program, effect on executive function, quality of life and depression, anxiety and stress: a randomized controlled trial
}

\author{
Ágata Vieira ${ }^{\mathrm{a}, \mathrm{b}}$, Cristina Melo ${ }^{\mathrm{b}}$, Jorge Machadoc ${ }^{c}$ and Joaquim Gabriel ${ }^{\mathrm{d}}$

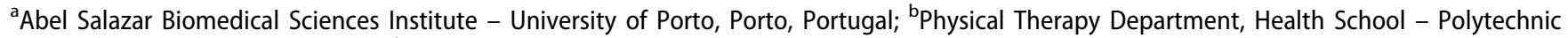 \\ Institute of Porto, Porto, Portugal; ' 'Laboratory of Applied Physiology, Abel Salazar Biomedical Sciences Institute - University of Porto, Porto, \\ Portugal; ${ }^{d}$ Mechanical Engineering Department - Automation, Instrumentation and Control, Faculty of Engineering - University of Porto, Porto, \\ Portugal
}

\begin{abstract}
Purpose: To analyse the effect of a six-month home-based phase III cardiac rehabilitation (CR) specific exercise program, performed in a virtual reality (Kinect) or conventional (booklet) environment, on executive function, quality of life and depression, anxiety and stress of subjects with coronary artery disease.

Methods: A randomized controlled trial was conducted with subjects, who had completed phase II, randomly assigned to intervention group 1 (IG1), whose program encompassed the use of Kinect $(n=11)$; or intervention group 2 (IG2), a paper booklet $(n=11)$; or a control group (CG), only subjected to the usual care $(n=11)$. The three groups received education on cardiovascular risk factors. The assessed parameters, at baseline (M0), 3 (M1) and 6 months (M2), were executive function, control and integration in the implementation of an adequate behaviour in relation to a certain objective, specifically the ability to switch information (Trail Making Test), working memory (Verbal Digit Span test), and selective attention and conflict resolution ability (Stroop test), quality of life (MacNew questionnaire) and depression, anxiety and stress (Depression, Anxiety and Stress Scale 21). Descriptive and inferential statistical measures were used, significance level was set at 05 .

Results: The IG1 revealed significant improvements, in the selective attention and conflict resolution ability, in comparison with the CG in the variable difference M0-M2 $(p=.021)$ and in comparison with the IG2 in the variable difference $M 1-M 2$ and $M 0-M 2(p=.001$ and $p=.002$, respectively). No significant differences were found in the quality of life, and depression, anxiety and stress.

Conclusions: The virtual reality format had improved selective attention and conflict resolution ability, revealing the potential of $C R$, specifically with virtual reality exercise, on executive function.
\end{abstract}

KEYWORDS Virtual reality exercise; cardiac rehabilitation; executive function; quality of life; depression; anxiety and stress

\section{Introduction}

When we talk about cardiovascular diseases, coronary artery disease is one of the most common diseases [1]. It is important to implement measures that work in both primary and secondary prevention, so, in this context, cardiac rehabilitation (CR) programs were developed [2]. The phase III, the last one of $C R$, focuses on long-term prevention, representing long-term outpatient supervision of patient adherence to prescribed lifestyle [3].

Cognitive decline might be associated with a reduction of the cardiovascular function, derived from the aging process, due to the progressive decline of oxygenation and hypoxia at the brain level $[4,5]$. As time elapses, the autorepair and autoadaptation (neuroplasticity) capacities of the nervous system are affected and become unable to compensate the lost neural networks, leading to a decrease in the neural tissues' density [4]. Several mechanisms have been singled out as contributions to the cognitive dysfunction in a patient with cardiovascular disease, including the low cardiac output, endothelial dysfunction, low physical activity and reduced blood flow to the brain [6]. With this, there is reason to believe that participation in CR programs can improve cognitive function in these patients [6]. In this study, we specifically explored the executive function, which consists of metacognitive capacities that open space to the control and integration in the implementation of an adequate behaviour in relation to a certain objective, that requires attention, programing and planning sequences, as well as inhibiting processes and contradictory information which are the responsibility of the pre-frontal cortex [7-9].

According to Antunes et al. [4], physical exercise improves and protects brain function and can fasten cognitive processing. 
Aerobic exercise improves not only cardiovascular fitness, but also brain function and cognition [10,11]. Physical exercise strengthens executive function by increasing neurogenesis and vascular plasticity [12]. Thus, it is often suggested that physically active subjects are in lesser risk of developing mental disorders than sedentary subjects [13]. Physical exercise is therefore essential to maximize our physical, psychological and social well-being by promoting the development of motor learning skills and the cognitive function, which influence our quality of life $[1,4]$.

It is recognized that cardiovascular diseases influence the quality of life of the individual, since it is related to an increase in functional dependence [14]. The presence of depressive symptoms has been associated, more and more, with a higher morbidity and mortality rate in cardiovascular diseases [15]. Since the recovery and/or maintenance of quality of life is one of the primary goals of CR [2] it becomes important to study its impact on quality of life and depression, anxiety and stress. There is sufficient evidence (class 1) that clarifies the importance of CR programs in improving health-related quality of life [14], so it is pertinent to its analysis in phase III of CR.

As far as CR is concerned, an alternative to the implementation of $C R$ programs are home-based $C R$ programs, given the growing economic burden of coronary artery disease in the whole world, the development of an affordable, acceptable and suitable CR method based on the community becomes increasingly important $[16,17]$, especially if we are talking about the phase III of CR. In addition, the use of information and communication technologies, Telehealth, might be a viable alternative in CR [18]. In this context, the possibility of using the Kinect, a new virtual reality-based technology of Xbox, Microsoft [19], as a working tool, can be extremely valuable. Virtual exercise programs, interactive games, are becoming increasingly popular in general rehabilitation, being the Kinect a possible tool, however, there is little research on the use of the Kinect as a therapeutic tool [19]. Real-time technology and Microsoft's motion sensor, Kinect, can make rehabilitation more successful and fun [20]. Kinect is inexpensive, easy to set up and can be used in clinical and home environments, being that this accessibility can facilitate rehabilitation [20]. The use of computers and gaming equipment in physical therapy is progressively more relevant in the medical community [19], so why not to explore this in a context of CR.

Considering all the aforementioned aspects that contribute to the decline of executive function, and the important impact of cardiovascular diseases in quality of life and depression, anxiety and stress, it becomes important to conduct this type of study in a CR context, phase III, especially if using new technologies. The goal of this study was to analyse the effect of a specific exercise program which was designed to be performed at home context during the phase III of $\mathrm{CR}$, over a six-month period. The study compared a virtual reality format (Kinect), a conventional format (booklet) and a control group (CG) (usual care) and measured changes in executive function, quality of life and depression, anxiety and stress, for subjects with coronary artery disease.

In the following sections it will be described in detail the study, with presentation of the sample and intervention with description of the exercise protocol and specifically the virtual reality exercise. It will also describe the data collection and present and discuss the results obtained.

\section{Methods}

This study is part of a global project and, in the same, presented a similar methodology to that previously published in Vieira et al. $[21,22]$, duly referenced throughout the present study.

\section{Study design}

This study is a prospective randomized controlled trial single site, according to Vieira et al. [22], using a three arm, parallel group over a 23-month period.

According to Vieira et al. [21,22], the study was approved by the Ethics Committee of the Centro Hospitalar do Porto (Porto Healthcare Centre in Portugal) - Teaching, Coaching and Research Department - N/REF. 212/12 (165-DEFI/157-CES) and by the Ethics Committee of the Health School, Polytechnic Institute of Porto - 1489/2012. According to Vieira et al. [21,22], all procedures were conducted according to the Declaration of Helsinki and, according to Vieira et al. [22], the study is registered at ClinicalTrials.gov (NCT02753829).

\section{Sample}

According to Vieira et al. [21,22], the sample was obtained from the Centro Hospitalar do Porto. According to Vieira et al. [21,22], the target population was composed of subjects who had just completed the phase II of CR at the Cardiovascular Prevention and Rehabilitation Unit. The subjects were, by the research coordinator at the end of the phase II, in-person and, according to Vieira et al. [22], individually invited to participate in this study. According to Vieira et al. [22], the enrolment and assignment were conducted by the research coordinator, with the support of the responsible of the Unit, according to the inclusion and exclusion criteria (Table 1) according to Vieira et al. [21,22].

The flow diagram, according to Vieira et al. [22], is presented in Figure 1. According to Vieira et al. [22], the participants were randomly assigned to one of the three groups: intervention group 1 (IG1) - a home-based CR program, using a computer and Kinect (virtual reality format) $(n=15)$; intervention group 2 (IG2) - a home-based CR program using a paper booklet (conventional format) $(n=15)$; and a CG, only subjected to the usual care $(n=16)$. According to Vieira et al. [22] a randomization by blocks was used, and an allocation sequence based on a fixed block size of 3

Table 1. Inclusion/exclusion criteria. The same as Vieira et al. [21,22].

\begin{tabular}{|c|c|}
\hline Inclusion criteria & Exclusion criteria \\
\hline $\begin{array}{l}\text { - Subjects of both sexes, aged between } 40-75 \text { years; } \\
\text { - Completed phase II of CR at the Cardiovascular Prevention and Rehabilitation } \\
\text { - Unit; } \\
\text { - Coronary artery disease, diagnosed and stabilized, with no unstable angina } \\
\text { and complex ventricular arrhythmias [23-26], with or without percutaneous } \\
\text { coronary intervention and with a final diagnosis of acute myocardial infarction } \\
\text { or stable angina pectoris; } \\
\text { - Access to a computer with Microsoft Windows } 7 \text { (minimum). }\end{array}$ & $\begin{array}{l}\text { - Heart surgery; } \\
\text { - Non-completed stress test due to maximum fatigue; } \\
\text { - Pregnancy or planning to get pregnant; } \\
\text { - Cardiovascular high risk }[23,25,26] \text { according to Pescatello et al. [27]; } \\
\text { - Pacemaker, severe neurological, musculoskeletal or pulmonary diseases, and } \\
\text { uncompensated metabolic disorders, reported dementia [25-27], cardiomyopa- } \\
\text { thies and previous cardiorespiratory arrest non-associated with acute myocar- } \\
\text { dial infarction or heart procedures; } \\
\text { - Significant and uncompensated visual [25] and auditory deficits; } \\
\text { - Uneducated and/or with no fluency in Portuguese; } \\
\text { - Attending or planning to attend gym or regular physical exercise programs. }\end{array}$ \\
\hline
\end{tabular}




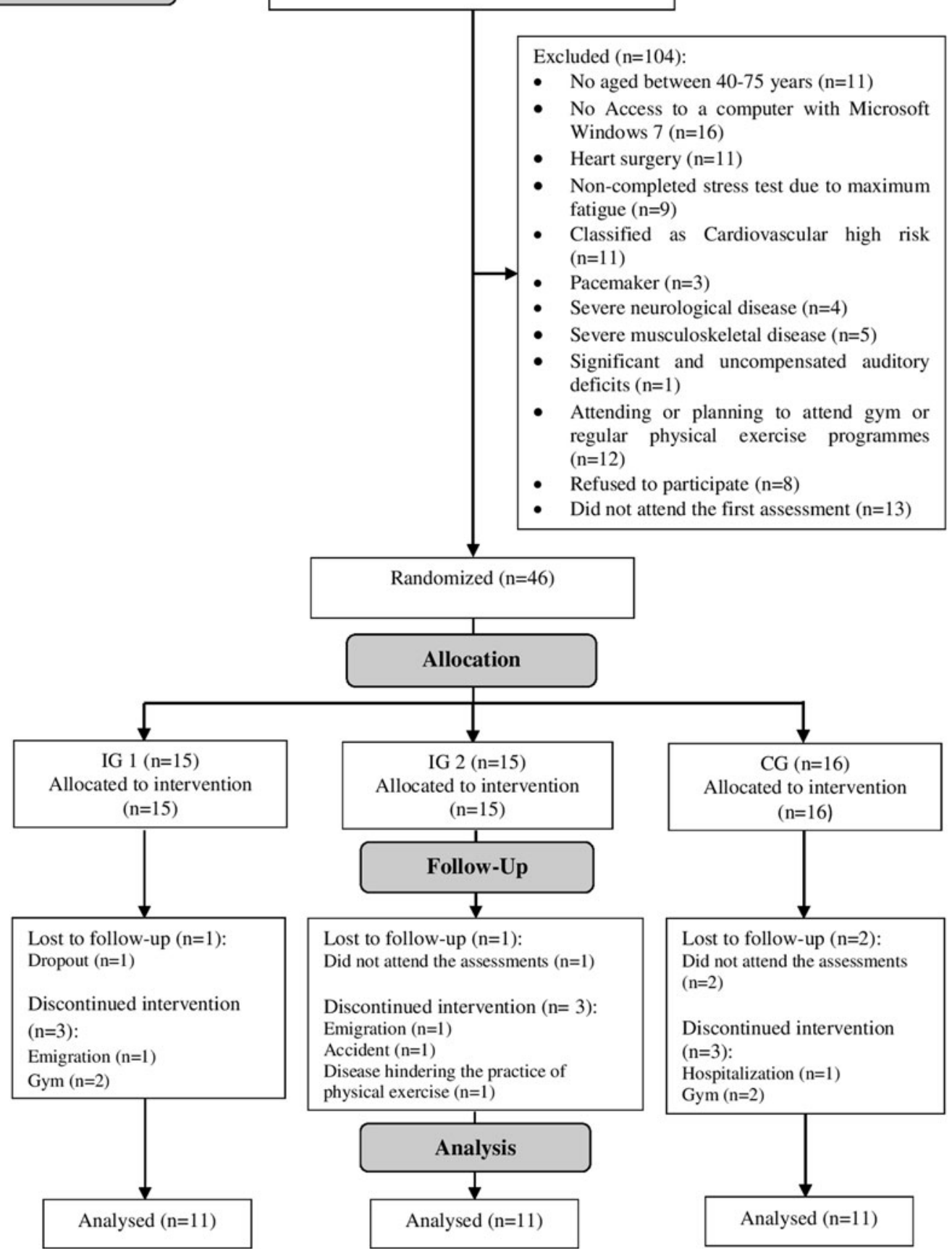

Figure 1. Flow diagram of patients (assessed for eligibility $n=150$ ). According to Vieira et al. [22]. CG: control group; IG1: intervention group 1; IG2: intervention group 2.

was generated with a computer random number generator by a blind investigator, not involved in the trial.

According to Vieira et al. [21,22] throughout the follow-up, four subjects were excluded from IG1 and, according to Vieira et al. [22], from IG2, and five from CG. Therefore, according to Vieira et al. [22], the final sample was composed of 33 subjects: IG1 $n=11$, IG2 $n=11$ and CG $n=11$.

\section{Intervention}

All participants of the three groups received education on cardiovascular risk factors. The intervention groups had also access to a specific exercise program, performed with the virtual reality (Kinect), IG1, or a paper booklet, IG2.

The teaching process and in-person follow-up took place at the Cardiovascular Prevention and Rehabilitation Unit, Health School of Porto and/or participant's home.

According to Vieira et al. [22], were first delivered pamphlets to all participants of the three groups with information on the risk factors for cardiovascular disease, which focused on eating habits, smoking and physical activity; the pamphlets were presented and questions regarding the pamphlets were answered. A leaflet with a brief presentation of the study, according to Vieira et al. [22], was also distributed. With regard to the intervention groups, according to Vieira et al. $[21,22]$, before moving on to the exercise 
Table 2. Presentation of the exercise protocol. The same as Vieira et al. [21,22].

\begin{tabular}{|c|c|c|}
\hline Session phase & Exercise & Description \\
\hline $\begin{array}{l}\text { Warm up } \\
10 \text { minutes }\end{array}$ & 1- Marching in place & $\begin{array}{l}\text { Hip flexion, below the waist level, with flexion of the contralateral glenohum- } \\
\text { eral joint, always in the same place. } \\
\text { After } 3 \text { months performing hip flexion up to the waist level. }\end{array}$ \\
\hline \multirow{6}{*}{$\begin{array}{l}\text { Workout } \\
\text { Strength } \\
20-25 \text { minutes (to each individual } \\
\text { repetitions calculated by } 65-70 \% \\
\text { of the HR reserve) }\end{array}$} & & \\
\hline & 2- Squats & $\begin{array}{l}\text { With feet shoulder width apart, perform knee flexion, without going over the } \\
\text { toes, with bilateral flexion of the glenohumeral joint to } 90^{\circ} \text {. } \\
\text { After } 3 \text { months performing two series with a one minute break. }\end{array}$ \\
\hline & 3- Crossing & $\begin{array}{l}\text { Keep marching in place throughout the exercise; performed the 1st proprio- } \\
\text { ceptive neuromuscular facilitation diagonal for bilateral upper limb flexion } \\
\text { (glenohumeral flexion, adduction and external rotation). } \\
\text { After } 3 \text { months performing two series with a one minute break, the 2nd pro- } \\
\text { prioceptive neuromuscular facilitation diagonal for bilateral upper limb flex } \\
\text { ion (glenohumeral flexion, abduction and external rotation). }\end{array}$ \\
\hline & 4-Ankle movement & $\begin{array}{l}\text { Dorsiflexion/plantar flexion of the ankles while standing. } \\
\text { After } 3 \text { months performing two series with a one minute break. }\end{array}$ \\
\hline & $\begin{array}{l}\text { 5- Backward movements } \\
\text { of the arms }\end{array}$ & $\begin{array}{l}\text { Keep marching in place throughout the exercise; perform extension, abduction } \\
\text { and external rotation of the glenohumeral for the complete range. At the } \\
\text { end of the movement forcefully increased range of movement } 10 \text { times. } \\
\text { After } 3 \text { months performing two series with a one minute break. }\end{array}$ \\
\hline & 6- Sit and stand & $\begin{array}{l}\text { Sitting in a chair with the upper limbs crossed over the chest. Sitting should } \\
\text { be performed in a controlled movement. } \\
\text { After } 3 \text { months cut down seat height. }\end{array}$ \\
\hline \multirow[t]{2}{*}{$\begin{array}{l}\text { Endurance } 35-45 \text { minutes (to each } \\
\text { individual repetitions calculated } \\
\text { by } 65-70 \% \text { of the HR reserve) }\end{array}$} & 7- Step forward, sideways and backward & $\begin{array}{l}\text { Perform forward and backward half-step with bilateral upper limb flexion, anc } \\
\text { sideways half-step with bilateral upper limb abduction and external rota- } \\
\text { tion. }\end{array}$ \\
\hline & 8- Walk (30 minutes) & $\begin{array}{l}\text { After } 3 \text { months performing two series with a one minute break. } \\
\text { After } 3 \text { months, if possible, increased to } 60 \text { minutes. }\end{array}$ \\
\hline \multirow[t]{2}{*}{ Stretching 6 minutes } & 9- Calf muscle stretching & $\begin{array}{l}\text { Stretch the triceps surae } \\
4 \text { repetitions/maintain } 15 \text { seconds }\end{array}$ \\
\hline & $\begin{array}{l}\text { 10- Anterior forearm muscle } \\
\text { stretching }\end{array}$ & $\begin{array}{l}\text { Stretch the wrist flexors } \\
4 \text { repetitions/maintain } 15 \text { seconds }\end{array}$ \\
\hline
\end{tabular}

HR: heart rate.

protocol and respective instructions, the subjects of the intervention groups attended three classes of teaching and demonstration (namely regarding the preparation of home space), with at least a one-day break between them $[25,26]$. IG1, according to Vieira et al. [21,22], was also taught on how to use Kinect. Heart rate (HR) training, for each participant, was determined by the research coordinator, according to Vieira et al. [21,22] using Karnoven's formula, with the $H R$ reserve, based on the maximum $H R$ of the stress test and obtaining the basal HR with the participant in a sitting and relaxed position. According to Vieira et al. [22], a Polar Wearlink Coded cardiofrequencimeter, model FT7 with watch, with an excellent precision (error of $\pm 1 \%$ or $\pm 1 \mathrm{bpm}$ ) [28] was used to determine the HR training, as well as the number of repetitions.

The exercise protocol, performed for the intervention groups during six months, according to Vieira et al. [21,22] was adapted to the characteristics of the home context in the form of a selfmonitoring system, presenting two progressive levels, so as to meet the principles of overcharge, specificity and reversibility, being performed at moderate intensity, at level 1 of the exercise protocol, the exercise intensity was $65 \%$ of the HR reserve $[27,29]$. According to Vieira et al. [21,22], three months passed, participants moved to level 2, with an intensity of $70 \% \mathrm{HR}$ reserve $[27,29]$. According to Vieira et al. [21,22], exercise progression was made by increasing the number of repetitions, series and/or with modifications in the way how the exercise was performed.

According to Vieira et al. [21,22], the exercise intensity and the number of repetitions were also monitored with the Borg scale of perceived exertion (ratings between 6 and 20), so as to achieve an interval between 12 and $13[26,27,29]$. According to Vieira et al. [22], the scale presents a criterion validity of $r=0.62$ when compared with HR and $r=0.64$ when compared with VO2 max
[30]. According to Vieira et al. [21,22], the exercise protocol was performed three times a week [1] over six months $[25,26]$ in the most suitable time for each participant. According to Vieira et al. [22], in addition, in the remaining days, a daily walk of 30 minutes was recommended [1].

According to Vieira et al. [21,22], the exercise protocol (Table 2), designed by a certified expert in Physical Therapy with five years of experience in the field and adapted from Noites et al. [31], was made up of 10 exercises: a warm up exercise; seven exercises of conditioning workout aimed at enhancing cardiorespiratory and muscular endurance and/or strength, and two exercises to increase limb flexibility. Additionally, according to Vieira et al. [21,22], exercises 1, 4, 6 and 7 were also aimed at improving balance, as well as exercise 5 and progression of exercise 3 were aimed at improving thoracic curve.

The exercise protocol was exactly the same with the virtual reality (Kinect), IG1, and the paper booklet, IG2.

According to Vieira et al. [22], IG2 performed the home-based program with paper booklets for consultation. IG1's program included the use of virtual reality exercise, according to Vieira et al. $[21,22]$ with the Kinect (Microsoft) and a computer, having the system been installed at each participant's home. According to Vieira et al. [21,22], the Kinect-RehabPlay project, developed in the Faculty of Engineering, University of Porto [32], relies on software to monitor and evaluate the rehabilitation exercises, which have to be performed by the user and captured by the Kinect sensor, providing him/her with real time feedback about the given challenge. [32]. According to Vieira et al. [21,22], this system provides a virtual physical therapist performing the exercise and providing indications concerning the quality of execution [32]. According to Vieira et al. [21,22], the participant is also represented as a second avatar, which interactively follows the physical 
therapist [32]. According to Vieira et al. [21,22], the software uses the Microsoft Kinect to track individual movement and making a match with a pre-defined pattern. According to Vieira et al. $[21,22]$, this feature monitored the number of repetitions for each exercise, according to the pre-calculated value, and set it to the individual exercise profile, being the same referenced in the program along with the respective exercise.

According to Vieira et al. [21], the Kinect-RehabPlay system is composed of three modules, the virtual reality environment, the Kinect sensor, and the monitoring software package, serving the Kinect sensor as an input to the virtual reality environment, which is monitored by the monitoring software package [32]. According to Vieira et al. [21], the exercises to be performed are presented in a graphical form to encourage the user to continue with the exercises, but also to demonstrate how to perform them, being the virtual environment in which it takes place an important part of the Kinect-RehabPlay system [32], offering the system visual and audio instructions.

Figure 2, according to Vieira et al [21], shows the interaction between the user and the Kinect-RehabPlay system, in the home of one of the participants.

Throughout the study, the subjects in IG1 and IG2, according to Vieira et al. [21,22], added, during the sessions, the HR values, Borg rating and eventual comments on an 'Exercise Diary' and in this way proving their assiduity to the exercises and so their adherence to the program. According to Vieira et al. [22], adherence percentage was so defined as the number of sessions attended, according to the registration in the "Exercise Diary", divided by the total number of sessions prescribed (three sessions a week during 6 months).

The registration of the HR training and Borg rating, at home during the sessions, worked as a way of monitoring. The participants assessed the HR with the aid of a cardiofrequencimeter, that they had voluntarily acquired, or with manual measurement previously taught.

The CG was only subjected to the usual care. According to Vieira et al. [22], equal to what happened with the intervention groups, they also received education on cardiovascular risk factors, having being the daily walks also encouraged.

For the three groups, according to Vieira et al. [21,22], phone contacts were scheduled for the weeks 4,10 and 22, as well as,

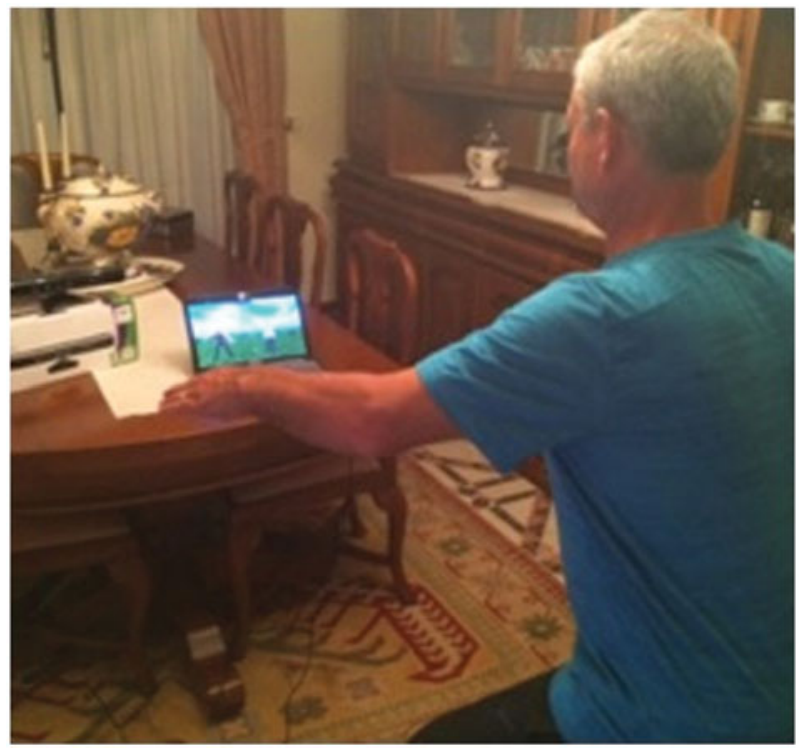

Figure 2. Virtual reality exercise in a real context. The same as Vieira et al. [21]. for the intervention groups, home visits or in-person meetings (aimed at reevaluating and readjusting the exercises) for weeks 6 and 18 [25,26]. According to Vieira et al. [21,22], emails and/or phone messages were sent for the participants of the intervention groups, on a weekly basis, emphasizing the importance of adhering to the program.

All participants, of the three groups, had access to the research coordinator contacts.

\section{Data collection}

According to Vieira et al. [22], a pilot study was conducted among 10 subjects whose characteristics resembled the ones from the target population, with the aim of assessing the feasibility of the exercises, the reliability of the instruments and to improve the time management of data collections.

The assessment of the study for the three groups, according to and adapted from Vieira et al. [22] encompassed three moments: a baseline/initial moment ( $\mathrm{M} 0$ ), right after the termination of the phase II and before the beginning of the program; an intermediate moment (M1), three months after the beginning of the program; and a final moment (M2), six months after the beginning of the program, being present in Figure 3 the time management of the study and respective data collections with the instruments. According to Vieira et al. [22], data collection took place at the Cardiovascular Prevention and Rehabilitation Unit and the Health School of Porto.

At M0, the participants, according to Vieira et al. [22], filled in a sample selection and characterization questionnaire.

This was made up of eight personal and demographic questions, nine questions regarding medical history and four regarding $C R$, namely the issue of attending gym or some regular physical exercise program, access to a computer and interest in participating in the study. The medical history and data, of each subject, were collected and/or checked in the clinical process.

The participants were enclosed in a quiet environment with good lighting, no sound or visual distractions and with all the commodities necessary to perform the tests. A Samsung chronometer was used for time account. At M0, the Montreal Cognitive Assessment (MoCA) was used as a brief and practical method to perform a primary cognitive screening, as it is an instrument which assesses different cognitive domains (attention and concentration, executive functions, memory, language, visuoconstructive abilities, conceptual thinking, calculations and orientation) [33]. The MoCA score is calculated by summing the points of the completed tasks successfully, in a range from 0 to 30 points [33]. According to Freitas et al. [33] this test, adapted to the Portuguese population, shows an internal consistency of Cronbach's $\alpha=.94$. At M0, M1 and M2, to assess executive function, one of the variables under study, specifically the ability to switch information, working memory and selective attention and conflict resolution ability were used the Trail Making Test (TMT), the Verbal Digit Span (VDS) test and the Stroop test, respectively.

With respect to TMT, the intra-observer reliability was good in the present pilot-study $(I C C=0.63)$ [34]. The TMT is a neuropsychological test to assess the ability to switch information $[5,7,35,36]$, individual's ability to switch between different tasks, or even between different elements of the same task, in order to test cognitive flexibility $[7,8]$. It is divided into two parts: TMT-A, in which participant connects 25 circles numbered from 1 to 25 , and TMT-B, in which participant connects circles numbered from 1 to 13 alternating with letters from $A$ to $L[5,7,35,36]$. The final score of each part depends on the time spent in the completion of the test [36]. The total score was calculated by subtracting part B 


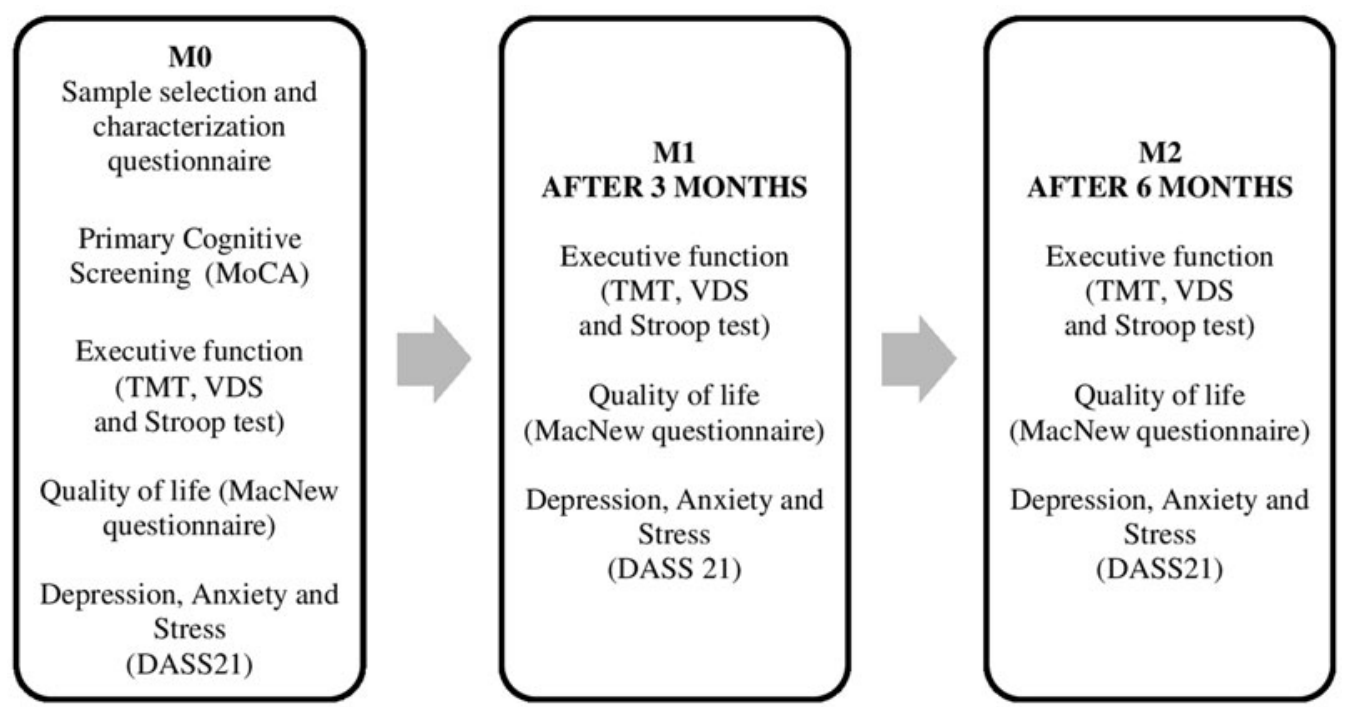

Figure 3. Time management of the study and respective data collections with the instruments. M0: baseline/initial moment; M1: intermediate moment ( 3 months); M2: final moment (6 months); DASS 21: Depression, Anxiety and Stress Scale 21; MoCA: Montreal Cognitive Assessment; TMT: Trail Making Test; VDS: Verbal Digit Span.

score from part A score, considering that the lower the score is, the bigger will be the capacity to switch information and so the cognitive flexibility of the participant [37].

Afterwards, the VDS test was used. This test is an instrument related to the working memory's central executive component, a process that allows to preserve the information required for a particular instant and remember it in the short term [38-40] and update replacing old information and not relevant by most recent and relevant $[7,41]$. The intra-observer reliability showed by this test in the present pilot study was good (ICC $=0.71)$ [34]. It is divided into two parts: VDS-Forward test, in which participant has to say sequences of numbers in the normal digit order, and VDSBackward test, in which participant has to say sequence of numbers in the inverse order. A score is attributed to each correct sequence; the maximum score for each part, VDS-Forward test and VDS-Backward test, is 14, and the minimum score is 0 [39]. The different scores of the two parts are used to calculate the total score of executive function's working memory, by subtracting the second part from the first part, knowing that the lower the difference between the results is, the better will be the performance of the participant [39].

Lastly, the Stroop test, was applied. This is a neuropsychological test aimed at assessing selective attention and conflict resolution ability [40], the monitoring of information relevant to the task you want to perform, ability to suppress a dominant or automatic response, when this proves inadequate, focusing the attention on what really intended and inhibiting another competitor information [7,8]. According to Esgalhado et al. [42] this is adapted to the Portuguese population with an internal consistency of Cronbach's $\alpha=.873$. The intra-observer reliability observed for this test in the present pilot study was good $(I C C=0.71)$ [34]. The score of this test is obtained from the number, in 45 seconds for each, of words (W) read on a page with 100 words with colour names (red, green, blue) printed in black ink; number of colours (C) listed on a page with 100 crosses printed in colours (red, green and blue); and number of items (colours) listed (PC) on a page with 100 words with colour names, but the name of the colour does not match with the colour in which the word is written. The total score was obtained by subtracting $P C$ from $\mathrm{PC}^{\prime}$ (estimated score: $\mathrm{PC}^{\prime}=\mathrm{P} \times \mathrm{C} / \mathrm{P}+\mathrm{C}$ ) [43]. The higher the total score is, the better will be the selective attention and conflict resolution ability [43].

At M0, M1 and M2 each participant completed, in self-administration, the MacNew questionnaire for the assessment of quality of life, as well as the Depression, Anxiety and Stress Scale 21 (DASS 21). According to Leal [44] and Tavares et al. [45], the MacNew Heart Disease Health-related Quality of Life questionnaire, validated to the Portuguese population and with a high internal consistency (Cronbach's $\alpha=.93$ ), was used to assess the quality of life related to the health condition upon heart disease, and comprises physical, emotional and social dimensions.

The MacNew total score is calculated using the mean of all items, while the score of each dimension is calculated by the mean of the respective items $[44,45]$. The intra-observer reliability perceived in the present pilot-study was excellent $($ ICC $=0.87$ ) [34].

The DASS 21 covers three dimensions - depression, anxiety and stress. The total score is calculated by summing the scores obtained in each dimension [46]. The intra-observer reliability in the present pilot-study was excellent $(I C C=0.90)$ [34]. According to Pais-Ribeiro et al. [46], in this instrument validated to the Portuguese population, the three dimensions must be assessed separately, being the internal consistency .85 for the depression dimension, .74 for the anxiety dimension and .81 for the stress dimension (Cronbach's $\alpha$ ).

\section{Statistics}

Assuming a power of $80 \%$ with a $5 \%$ significance level, considering the one-way analysis of variance (ANOVA) test, the power calculation revealed a training effect of 0.76 and 0.72 on the Stroop test total score indicating a need for 21 and 24 participants to ensure statistical power to detect differences between the three groups in the variable difference $M 1-M 2$ and $M 0-M 2$, respectively.

The statistical analysis was accomplished using the IBM SPSS 22 software (IBM Corp., Armonk, NY) for Windows, with a significance level of .05 and a confidence interval of 95\% [47].

The sample was characterized using descriptive statistics. In $\mathrm{MO}$, in the variables of the sample characterization, whenever the distribution was normal, the one-way ANOVA test was used for 
the rational and nominal variables, and whenever the distribution was not normal, the Kruskal-Wallis test and the Fisher test for independent samples were used for the rational and nominal variables, respectively. The $t$ test for independent samples was used to compare adherence rates between the intervention groups, since the variable followed the normal pattern [47].

In the intra-group analysis of the TMT, VDS test and Stroop test total scores, to compare M0, M1 and M2, the ANOVA test for repeated measures and the Bonferroni post hoc test were used, since the variables followed the normal pattern. In the intra-group analysis of the MacNew questionnaire and DASS 21 total scores and dimensions, to compare the $\mathrm{M} 0, \mathrm{M} 1$ and $\mathrm{M} 2$, the ANOVA test for repeated measures and the Bonferroni post hoc test or the Friedman test and the Dunn post hoc test were used, respectively, in case the distribution was normal or not [47].

In the inter-group analysis for the VDS test and Stroop test total scores, in the several moments (M0, M1 and M2) and in the variable difference between the different assessment moments $(M 0-M 1, M 1-M 2$ and $M 0-M 2)$, and for the TMT total score in MO were used the one-way ANOVA test and the Tukey post hoc test since the variables followed a normal pattern. For the TMT total score, $M 1$ and $M 2$ and the variable difference between the different assessment moments (M0 - M1, M1 - M2 and M0 - M2), the $t$ test for independent samples was used for the analysis between two groups since the variables followed a normal pattern. In the inter-group analysis for the MacNew questionnaire and DASS 21, total scores and dimensions, in the several moments (M0, M1 and M2) and in the variable difference between the different assessment moments (M0-M1, M1-M2 and $M 0-M 2)$, whenever the distribution was normal, the one-way ANOVA test and the Tukey post hoc test were used, and whenever the distribution was not normal the Kruskal-Wallis test and the Dunn post hoc test were used. In the inter-group analysis of the dimension depression of the DASS 21, in M1, M2 and variable difference $(M 0-M 1, M 1-M 2$ and $M 0-M 2)$, were used the $t$ test for independent samples and the Mann-Whitney test for the analysis between two groups in case the distribution was normal or not, respectively [47].

\section{Results}

According to Vieira et al. [22], the final sample was composed of 33 subjects, all men. At M0, in the MoCA, seven participants from IG1 were below the normal value (26), whereas in IG2 and CG this was true for every participant. According to Table 3, at M0, the most frequent cardiovascular risk factors were Dyslipidemia, Hypertension and Smoking in all three groups and Obesity in CG, most of the participants were taking Blood Platelet Antiaggregant, Beta blockers and Statins and, in the three groups, the vast majority of subjects presented low cardiovascular risk.

According to Vieira et al. [22], at M0, no significant differences were found between the three groups $(p>05)$ in the sample characteristics (demographic and clinical characteristics, MoCA and medication) (Table 3). According to Vieira et al. [22], no significant differences were also found between the three groups $(p>.05)$ in the medication change throughout the study.

According to Vieira et al. [21,22] concerning the percentage of subjects adhering to the program, for three sessions a week, IG1 presented a mean of $82 \%$ in the first three months and $70 \%$ in the last three, with a mean of $77 \%$ over the six-month period. According to Vieira et al. [22], IG2 presented a mean of $90 \%$ in the first three months and $75 \%$ in the last three, with a mean of $83 \%$ for the whole six months. According to Vieira et al. [22], no significant differences were found between the two groups.
Table 3. Sample characteristics in M0. According to Vieira et al. [21,22].

\begin{tabular}{|c|c|c|c|}
\hline Variable & IG1 $(n=11)$ & $\mathrm{IG} 2(n=11)$ & CG $(n=11)$ \\
\hline Age (years) & $55 \pm 9.0$ & $59 \pm 11.3$ & $59 \pm 5.8$ \\
\hline $\mathrm{MoCA}$ & $25.0 \pm 1.7$ & $23.2 \pm 3.5$ & $24.5 \pm 1.4$ \\
\hline \multicolumn{4}{|l|}{ Professional situation } \\
\hline Active & $7(64 \%)$ & $2(1 \%)$ & $5(45 \%)$ \\
\hline Inactive & $4(36 \%)$ & $9(82 \%)$ & $6(55 \%)$ \\
\hline \multicolumn{4}{|l|}{ Reason for hospitalization } \\
\hline ACS without ST elevation & $6(55 \%)$ & $6(55 \%)$ & $5(45 \%)$ \\
\hline ACS with ST elevation & $5(45 \%)$ & $3(27 \%)$ & $6(55 \%)$ \\
\hline $\begin{array}{l}\text { Stable Angina Pectoris and } \\
\text { post-angioplasty }\end{array}$ & 0 & $2(18 \%)$ & 0 \\
\hline \multicolumn{4}{|l|}{ Cardiovascular risk factors } \\
\hline Dyslipidemia & $10(91 \%)$ & $9(82 \%)$ & $8(73 \%)$ \\
\hline Obesity & $2(18 \%)$ & $2(18 \%)$ & $4(36 \%)$ \\
\hline Diabetes Mellitus & $2(18 \%)$ & $3(27 \%)$ & $1(9 \%)$ \\
\hline Hypertension & $5(45 \%)$ & $6(55 \%)$ & $8(73 \%)$ \\
\hline Smoking & $5(45 \%)$ & $5(45 \%)$ & $4(36 \%)$ \\
\hline Family history & $1(9 \%)$ & $1(9 \%)$ & $2(18 \%)$ \\
\hline \multicolumn{4}{|l|}{ Pharmacology } \\
\hline Blood platelet & $9(82 \%)$ & $11(100 \%)$ & $10(91 \%)$ \\
\hline Antiaggregants beta blockers & $8(73 \%)$ & $9(82 \%)$ & $8(73 \%)$ \\
\hline Statins & $9(82 \%)$ & $11(100 \%)$ & $11(100 \%)$ \\
\hline Antihypertensive drugs & $4(36 \%)$ & $4(36 \%)$ & $6(55 \%)$ \\
\hline Vasodilators & $1(9 \%)$ & $3(27 \%)$ & $5(45 \%)$ \\
\hline Calcium channel blockers & 0 & $1(9 \%)$ & $1(9 \%)$ \\
\hline \multicolumn{4}{|l|}{ Cardiovascular risk } \\
\hline Low & $7(64 \%)$ & $7(64 \%)$ & $8(73 \%)$ \\
\hline Moderate & $4(36 \%)$ & $4(36 \%)$ & $3(27 \%)$ \\
\hline
\end{tabular}

ACS: acute coronary syndrome; CG: control group; IG1: intervention group 1; IG2: intervention group 2; MoCA: Montreal Cognitive Assessment.

Data are expressed as mean values and standard deviation or $n$ (\%). The cardiovascular risk was classified according to Pescatello et al. [27].

Table 4. Inter-group analysis at different moments of the TMT total score.

\begin{tabular}{|c|c|c|c|c|}
\hline Variable & Group & $\begin{array}{c}\mathrm{MO} \\
X \pm \mathrm{SD}\end{array}$ & $\begin{array}{c}M 1 \\
X \pm S D\end{array}$ & $\begin{array}{c}\mathrm{M} 2 \\
X \pm \mathrm{SD}\end{array}$ \\
\hline \multirow[t]{6}{*}{ TMT total score } & IG1 & $\begin{array}{c}64.9 \pm 29.0 \\
(n=11)\end{array}$ & $\begin{array}{c}51.1 \pm 15.2 \\
(n=11)\end{array}$ & $\begin{array}{c}44.5 \pm 17.1 \\
(n=11)\end{array}$ \\
\hline & IG2 & $\begin{array}{c}105.5 \pm 41.7 \\
(n=11)\end{array}$ & - & - \\
\hline & CG & $\begin{array}{c}51.2 \pm 32.3 \\
(n=11)\end{array}$ & $\begin{array}{c}47.4 \pm 17.8 \\
(n=10)\end{array}$ & $\begin{array}{c}53.0 \pm 29.8 \\
(n=11)\end{array}$ \\
\hline & $\begin{array}{l}F \\
p\end{array}$ & $\begin{array}{l}7.255 \\
0.003^{\mathrm{a}}\end{array}$ & NS & NS \\
\hline & Post hoc & $\begin{array}{c}\mathrm{IG} 1<\mathrm{IG} 2 \\
p=.027^{\mathrm{b}}\end{array}$ & - & - \\
\hline & & $\begin{array}{l}\mathrm{IG} 2>C \mathrm{CG} \\
p=.003^{\mathrm{b}}\end{array}$ & - & - \\
\hline
\end{tabular}

CG: control group; IG1: intervention group 1; IG2: intervention group 2; M0: baseline/initial moment; $M 1$ : intermediate moment (3 months); $M 2$ : final moment (6 months); NS: non-significant; TMT: Trail Making Test.

Data are presented as mean values $(X)$ and standard deviation (SD).

${ }^{a} p$ Values with the ANOVA test.

${ }^{\mathrm{b}} p$ Values for Tukey's post hoc test.

Table 5. Inter-group analysis at different moments of the VDS test total score.

\begin{tabular}{llccc}
\hline & & M0 & M1 & M2 \\
Variable & Group & $X \pm S D$ & $X \pm S D$ & $X \pm S D$ \\
\hline VDS test total score & IG1 & $1.9 \pm 1.3$ & $1.8 \pm 1.3$ & $2.2 \pm 1.3$ \\
& & $(n=11)$ & $(n=11)$ & $(n=11)$ \\
& IG2 & $1.5 \pm 1.3$ & $1.6 \pm 1.0$ & $1.8 \pm 1.0$ \\
& & $(n=11)$ & $(n=11)$ & $(n=11)$ \\
& CG & $2.1 \pm 1.1$ & $1.5 \pm 1.8$ & $1.4 \pm 1.1$ \\
& & $(n=11)$ & $(n=10)$ & $(n=11)$ \\
& $p$ & NS & NS & NS \\
\hline
\end{tabular}

CG: control group; IG1: intervention group 1; IG2: intervention group 2; M0: baseline/initial moment; $M 1$ : intermediate moment (3 months); $M 2$ : final moment (6 months); NS: non-significant; VDS: Verbal Digit Span.

Data are presented as mean values $(X)$ and standard deviation (SD). 
Table 6. Inter-group analysis at different moments and the variable difference of the Stroop test total score.

\begin{tabular}{|c|c|c|c|c|c|c|c|}
\hline \multirow[b]{2}{*}{ Variable } & \multirow[b]{2}{*}{ Group } & \multirow[b]{2}{*}{$\begin{array}{c}\mathrm{M} 0 \\
X \pm \mathrm{SD}\end{array}$} & \multirow[b]{2}{*}{$\begin{array}{c}\mathrm{M} 1 \\
X \pm \mathrm{SD}\end{array}$} & \multirow[b]{2}{*}{$\begin{array}{c}\mathrm{M} 2 \\
X \pm S D\end{array}$} & \multicolumn{3}{|c|}{ Variable difference } \\
\hline & & & & & $\begin{array}{c}\text { M0-M1 } \\
X\end{array}$ & $\begin{array}{c}\mathrm{M} 1-\mathrm{M} 2 \\
x\end{array}$ & $\begin{array}{c}\mathrm{M} 0-\mathrm{M} 2 \\
x\end{array}$ \\
\hline \multirow[t]{6}{*}{ Stroop test total score } & IG1 & $\begin{array}{c}-4.7 \pm 8.8 \\
(n=11)\end{array}$ & $\begin{array}{c}-4.0 \pm 7.8 \\
(n=11)\end{array}$ & $\begin{array}{l}1.7 \pm 7.1 \\
(n=11)\end{array}$ & $\begin{array}{c}0.7 \\
(n=11)\end{array}$ & $5.7(n=11)$ & $6.4(n=11)$ \\
\hline & IG2 & $\begin{array}{l}2.8 \pm 8.0 \\
(n=11)\end{array}$ & $\begin{array}{l}3.6 \pm 6.8 \\
(n=11)\end{array}$ & $\begin{array}{c}-0.9 \pm 6.8 \\
(n=11)\end{array}$ & $\begin{array}{c}0.8 \\
(n=11)\end{array}$ & $-4.5(n=11)$ & $-3.7(n=11)$ \\
\hline & CG & $\begin{array}{c}-1.3 \pm 8.0 \\
(n=11)\end{array}$ & $\begin{array}{c}-2.6 \pm 8.7 \\
(n=10)\end{array}$ & $\begin{array}{c}-2.4 \pm 5.7 \\
(n=11)\end{array}$ & $\begin{array}{c}-1.9 \\
(n=10)\end{array}$ & $0.8(n=10)$ & $-1.1(n=11)$ \\
\hline & $F$ & NS & NS & NS & NS & 9.265 & 7.891 \\
\hline & $p$ & - & - & - & - & $0.001^{\mathrm{a}}$ & $0.002^{\mathrm{a}}$ \\
\hline & Post-hoc & - & - & - & - & $\begin{array}{c}\text { IG1 \# IG2 } \\
p=.001^{\mathrm{b}}\end{array}$ & $\begin{array}{c}\text { IG1 \# IG2 } \\
p=.002^{\mathrm{b}} \\
\mathrm{IG} 1 \# \text { CG } \\
p=.021^{\mathrm{b}}\end{array}$ \\
\hline
\end{tabular}

CG: control group; IG1: intervention group 1; IG2: intervention group 2; M0: baseline/initial moment; M1: intermediate moment (3 months); M2: final moment (6 months); NS: non-significant.

Data are presented as mean values $(X)$ and standard deviation (SD).

${ }^{a} p$ Values with the ANOVA test.

${ }^{b} p$ Values for Tukey's post hoc test.

Table 7. Inter-group analysis at different moments of the Macnew questionnaire.

\begin{tabular}{|c|c|c|c|c|c|}
\hline Variable & & Group & $\begin{array}{c}\mathrm{M} 0 \\
X \pm \mathrm{SD}\end{array}$ & $\begin{array}{c}\mathrm{M} 1 \\
X \pm S D\end{array}$ & $\begin{array}{c}M 2 \\
X \pm S D\end{array}$ \\
\hline \multirow[t]{4}{*}{ MacNew questionnaire } & Total & $\begin{array}{c}\text { IG1 } \\
\text { IG2 } \\
\text { CG } \\
p\end{array}$ & $\begin{array}{c}5.7 \pm 1.0 \\
(n=11) \\
5.7 \pm 0.7 \\
(n=11) \\
5.9 \pm 0.6 \\
(n=11) \\
\text { NS }\end{array}$ & $\begin{array}{c}6.2 \pm 0.5 \\
(n=10) \\
6.0 \pm 0.7 \\
(n=11) \\
5.9 \pm 0.9 \\
(n=10) \\
\text { NS }\end{array}$ & $\begin{array}{c}6.2 \pm 0.8 \\
(n=11) \\
5.8 \pm 1.0 \\
(n=11) \\
6.0 \pm 0.6 \\
(n=11) \\
\text { NS }\end{array}$ \\
\hline & Physical & $\begin{array}{c}\mathrm{IG} 1 \\
\mathrm{IG} 2 \\
\mathrm{CG} \\
p\end{array}$ & $\begin{array}{c}5.7 \pm 1.0 \\
(n=11) \\
5.5 \pm 0.9 \\
(n=11) \\
5.8 \pm 0.8 \\
(n=11) \\
\quad N S\end{array}$ & $\begin{array}{c}6.0 \pm 0.8 \\
(n=11) \\
5.9 \pm 0.9 \\
(n=11) \\
5.8 \pm 1.1 \\
(n=10) \\
\text { NS }\end{array}$ & $\begin{array}{c}6.2 \pm 0.8 \\
(n=11) \\
5.7 \pm 1.2 \\
(n=11) \\
6.0 \pm 0.8 \\
(n=11) \\
\text { NS }\end{array}$ \\
\hline & Emotional & $\begin{array}{c}\text { IG1 } \\
\text { IG2 } \\
\text { CG } \\
p\end{array}$ & $\begin{array}{c}5.6 \pm 0.9 \\
(n=11) \\
5.4 \pm 0.7 \\
(n=11) \\
5.6 \pm 0.7 \\
(n=11) \\
\text { NS }\end{array}$ & $\begin{array}{c}5.8 \pm 0.8 \\
(n=11) \\
5.9 \pm 0.8 \\
(n=11) \\
5.6 \pm 1.1 \\
(n=10) \\
\text { NS }\end{array}$ & $\begin{array}{c}6.0 \pm 0.9 \\
(n=11) \\
5.8 \pm 1.1 \\
(n=11) \\
5.9 \pm 0.8 \\
(n=11) \\
\text { NS }\end{array}$ \\
\hline & Social & $\begin{array}{l}\text { IG1 } \\
\text { IG2 } \\
\text { CG }\end{array}$ & $\begin{array}{c}6.2 \pm 1.1 \\
(n=11) \\
6.0 \pm 1.0 \\
(n=11) \\
6.5 \pm 0.6 \\
(n=11) \\
\text { NS }\end{array}$ & $\begin{array}{c}6.5 \pm 0.6 \\
(n=11) \\
6.4 \pm 0.7 \\
(n=11) \\
6.7 \pm 0.3 \\
(n=9) \\
\mathrm{NS}\end{array}$ & $\begin{array}{c}6.6 \pm 0.8 \\
(n=11) \\
6.1 \pm 1.1 \\
(n=11) \\
6.6 \pm 0.6 \\
(n=11) \\
\text { NS }\end{array}$ \\
\hline
\end{tabular}

CG: control group; IG1: intervention group 1; IG2: intervention group 2; M0: baseline/initial moment; $\mathrm{M} 1$ : intermediate moment (3 months); $\mathrm{M2}$ : final moment (6 months); NS: non-significant.

Data are presented as mean values $(X)$ and standard deviation (SD).

For all the variables under study, no significant differences were found between the three groups at M0, except in the total score of the TMT $(F=7.255, p=.003)$ between IG1 and IG2 $(p=.027)$ and IG2 and CG $(p=.003)$ (Table 4$)$, as well as in the dimension depression of the DASS $21(F=5.133, p=.013)$ between IG1 and IG2 ( $p=.012)$ (Table 8). These variables, between these groups, were not subjected to subsequent analyses.

With regard to intra-group analysis in the TMT total score (ability to switch information), some significant differences were observed in the analysis of IG2 $(F=5.730, p=.011)$, with a significant decrease from M0 to M1 $(p=.042)$ and M0 to $M 2$ $(p=.033)$, representing an increase in the ability to switch information.

In the intra-group analyses of the VDS test total score (working memory), no significant differences were found however, in the intra-group analysis of the Stroop test total score it was possible to observe significant differences in IG1 $(F=5.491, p=.013)$, with a significant increase from $\mathrm{M} 1$ to $\mathrm{M} 2(p=.009)$ representing an increase in the selective attention and conflict resolution ability.

Still in the intra-group analyses, in the MacNew questionnaire to assess quality of life, IG1 showed significant differences in its total score $\left(X^{2}=6.889, p=.032\right)$, with a significant increase from M0 to M2 ( $p=.042)$, meaning an increase in quality of life. IG2 presented some significant differences in the emotional $(F=4.278$, $p=.028)$ and social dimensions $(F=5.752, p=.011)$, with a significant increase from M0 to $\mathrm{M} 1$ ( $p=.010$ and $p=.023$, respectively). Finally, as far as the DASS 21 is concerned, to assess depression, anxiety and stress, no significant differences were observed in any dimension or total score in the intra-group analyses.

Regarding the inter-group analyses, as stated above, in relation to the TMT total score (ability to switch information) there were significant differences between the groups at $\mathrm{MO}$, not being equal in terms of comparison. The only comparable groups were IG1 with CG however, no significant differences were found in their analyses in the remaining moments (Table 4). Also in the analysis of the variable difference (M0 - M1, M1 - M2 and $M 0-M 2)$ of the TMT total score, there were no significant differences between the comparable groups.

In the VDS test total score (working memory), no significant differences were found in the analysis between the three groups in the different moments (Table 5). In the analysis of the variable difference $(M 0-M 1, M 1-M 2$ and $M 0-M 2)$ of the VDS test total score, there were also no significant differences between the three groups.

The Stroop test total score showed significant differences between the groups in the variable difference $M 1-M 2$ $(F=9.265, p=.001)$, with a significant increase in IG1 in comparison with IG2 $(p=.001)$ and in the variable difference M0-M2 $(F=7.891, p=.002)$ with a significant increase in IG1 in comparison with CG $(p=.021)$ and in comparison with IG2 $(p=.002)$ (Table 6), representing an increase in the selective attention and conflict resolution ability in IG1 in comparison with IG2 and CG. 
Table 8. Inter-group analysis at different moments of the DASS 21.

\begin{tabular}{|c|c|c|c|c|c|}
\hline Variable & & Group & $\begin{array}{c}\mathrm{M} 0 \\
X \pm \mathrm{SD}\end{array}$ & $\begin{array}{c}\mathrm{M} 1 \\
X \pm S D\end{array}$ & $\begin{array}{c}\mathrm{M} 2 \\
X \pm S D\end{array}$ \\
\hline \multirow[t]{18}{*}{ DASS 21} & Total & IG1 & $\begin{array}{c}24.6 \pm 29.3 \\
(n=11)\end{array}$ & $\begin{array}{c}16.0 \pm 18.4 \\
(n=11)\end{array}$ & $\begin{array}{c}15.3 \pm 19.8 \\
(n=11)\end{array}$ \\
\hline & & IG2 & $\begin{array}{c}23.2 \pm 15.0 \\
(n=10)\end{array}$ & $\begin{array}{c}17.8 \pm 16.2 \\
(n=11)\end{array}$ & $\begin{array}{c}19.5 \pm 20.7 \\
(n=11)\end{array}$ \\
\hline & & CG & $\begin{array}{c}24.6 \pm 17.2 \\
(n=11)\end{array}$ & $\begin{array}{c}23.6 \pm 22.5 \\
(n=10)\end{array}$ & $\begin{array}{c}21.6 \pm 19.7 \\
(n=11)\end{array}$ \\
\hline & & $p$ & NS & NS & NS \\
\hline & Depression & IG1 & $\begin{array}{c}2.2 \pm 2.5 \\
(n=9)\end{array}$ & $\begin{array}{l}5.3 \pm 7.1 \\
(n=11)\end{array}$ & $\begin{array}{l}2.4 \pm 3.6 \\
(n=10)\end{array}$ \\
\hline & & IG2 & $\begin{array}{l}8.6 \pm 6.1 \\
(n=10)\end{array}$ & $\begin{array}{l}5.3 \pm 7.1 \\
(n=11)\end{array}$ & $\begin{array}{l}5.6 \pm 6.7 \\
(n=11)\end{array}$ \\
\hline & & CG & $\begin{array}{l}4.2 \pm 3.8 \\
(n=10)\end{array}$ & $\begin{array}{l}5.8 \pm 6.9 \\
(n=10)\end{array}$ & $\begin{array}{l}5.5 \pm 5.4 \\
(n=11)\end{array}$ \\
\hline & & $\mathrm{F}$ & 5.133 & & \\
\hline & & $p$ & $0.013^{a}$ & NS & NS \\
\hline & & Post-hoc & $\begin{array}{l}\mathrm{IG} 1<\mathrm{IG} 2 \\
p=0.012^{\mathrm{b}}\end{array}$ & & \\
\hline & Anxiety & IG1 & $\begin{array}{c}2.7 \pm 2.0 \\
(n=9)\end{array}$ & $\begin{array}{l}1.4 \pm 2.1 \\
(n=10)\end{array}$ & $\begin{array}{c}0.9 \pm 1.1 \\
(n=9)\end{array}$ \\
\hline & & IG2 & $\begin{array}{l}8.0 \pm 9.1 \\
(n=11)\end{array}$ & $\begin{array}{l}4.7 \pm 2.6 \\
(n=11)\end{array}$ & $\begin{array}{l}5.2 \pm 5.6 \\
(n=10)\end{array}$ \\
\hline & & CG & $\begin{array}{l}6.9 \pm 7.4 \\
(n=11)\end{array}$ & $\begin{array}{l}5.0 \pm 5.8 \\
(n=10)\end{array}$ & $\begin{array}{l}4.4 \pm 4.5 \\
(n=11)\end{array}$ \\
\hline & & $p$ & NS & NS & NS \\
\hline & Stress & IG1 & $\begin{array}{c}11.1 \pm 12.0 \\
(n=11)\end{array}$ & $\begin{array}{l}8.5 \pm 9.1 \\
(n=11)\end{array}$ & $\begin{array}{l}8.2 \pm 9.1 \\
(n=11)\end{array}$ \\
\hline & & IG2 & $\begin{array}{c}11.6 \pm 11.2 \\
(n=11)\end{array}$ & $\begin{array}{l}7.8 \pm 7.8 \\
(n=11)\end{array}$ & $\begin{array}{l}8.7 \pm 9.3 \\
(n=11)\end{array}$ \\
\hline & & CG & $\begin{array}{c}12.0 \pm 7.6 \\
(n=11)\end{array}$ & $\begin{array}{c}12.8 \pm 12.3 \\
(n=10)\end{array}$ & $\begin{array}{c}11.8 \pm 11.3 \\
(n=11)\end{array}$ \\
\hline & & $p$ & NS & NS & NS \\
\hline
\end{tabular}

CG: control group; DASS 21: Depression: Anxiety and Stress Scale 21; IG1: intervention group 1; IG2: intervention group 2; M0: baseline/initial moment; M1: intermediate moment (3 months); M2: final moment (6 months); NS: nonsignificant.

Data are presented as mean values $(X)$ and standard deviation (SD).

${ }^{a} p$ Values with the ANOVA test.

${ }^{\mathrm{b}} p$ Values for Tukey's post hoc test.

Regarding the MacNew questionnaire and DASS 21, no significant differences were found in the analysis between the three groups in any of the dimensions and total scores in the different moments (Tables 7 and 8, respectively) and in the analysis of the variable difference ( $M 0-M 1, M 1-M 2$ and $M 0-M 2)$.

As far as the dimension depression of the DASS 21, there were significant differences between the groups at M0, not being equal in terms of comparison. The only comparable groups were IG1 with CG and IG2 with CG however, no significant differences were found in their analyses in the remaining moments (Table 8). Also in the analysis of the variable difference $(M 0-M 1, M 1-M 2$ and $M 0-M 2)$, of the dimension depression of the DASS 21, there were no significant differences between the comparable groups.

It should be highlighted that, in the Stroop test and DASS 21, in the three groups and at the different moments, sometimes in the case of the DASS 21 and always in the Stroop test, the standard deviation was superior to the mean, presumably due to the expected interpersonal variability, taking into account the target variables of the analysis.

\section{Discussion}

The results of this study suggest that, in this sample, the integration in a specific exercise program at the phase III of CR based on the use of virtual reality exercise with the Kinect resulted in improvements in executive function, specifically in the selective attention and conflict resolution ability. These results highlight the virtual reality technology, but also the importance of performing the phase III of CR not only with the goal of keeping the results obtained in phase II. The Kinect, and so the virtual reality exercise, can be a tool to explore in CR. According to Chang et al. [20], the Kinect is cheap, easy to configure and can be used in home environments, so this accessibility can facilitate rehabilitation. Kim et al. [48] has noted that there are several recent studies that have pointed out that the $C R$ programs work in reducing the decline of executive function. Thus justified the importance of assessing this variable in a CR context and in particular in phase III, in order to evaluate the potential of this phase in executive function.

Despite the fact that no significant differences were observed between the groups in the results of the TMT total score, the group subjected to the conventional format presented, between the baseline/initial moment and the intermediate moment (3 months) and the baseline/initial and final ( 6 months) moments of the study, a significant decrease of the score, which means an increase in the ability to switch information and therefore cognitive flexibility, in other words the individual's ability to switch between different tasks, or even between different elements of the same task $[7,8]$. The total score of the VDS test, which assesses the working memory, a process that allows to preserve the information required for a particular instant and remember it in the short term [38-40] and update replacing old information and not relevant by most recent and relevant $[7,41]$ did not reveal significant differences, which means that this component of executive function was not influenced by the physical exercise, at least within this sample and with this exercise program.

Concerning the selective attention and conflict resolution ability, the ability of focusing the attention on what really intended and inhibiting another competitor information $[7,8]$ assessed by the Stroop test total score, the group inserted in the virtual reality format registered significant improvements in the last 3 months with significant improvements when compared with the $C G$, between the baseline/initial and final ( 6 months) moments of the study, and conventional format, between intermediate ( 3 months) and final (6 months) moments and the baseline/initial and final (6 months) moments of the study. This evincing a higher resistance of the participants to interference, and therefore better selective attention and conflict resolution ability, as stated by Esgalhado et al. [43]. The inhibition is fundamental for conflict management, as it blocks irrelevant information that obstructs the attention process [49].

The MoCA, used in this study as a primary cognitive screening, can prove the importance of using instruments to assess executive function in this sample, since the mean score at the baseline/initial moment, in the three groups, was lower than 26, which is considered the normal value [33]. According to Kramer et al. [50], moderate levels of physical activity are beneficial to the cognitive processes in middle-aged and elderly subjects. This proves the importance of implementing this type of programs in the phase III of $\mathrm{CR}$, especially considering that this phase focuses, according to Piepoli et al. [3], on a long-term approach. As has been said previously, physical exercise can explain the results obtained, by the evolution through neovascularization and creation of new synaptic networks, where a trophic effect is developed in the brain areas responsible for the sensory and motor function, and also by the optimization of the neurotransmitter function $[1,4,40]$ and increase of vascular plasticity [12].

As for the quality of life, and depression, anxiety and stress, it is possible that the obtained results might not have been influenced by other variables which can affect as the professional situation and cardiovascular risk factors, since the groups were comparable at the beginning of the study [14]. 
As far as the MacNew questionnaire is concerned (quality of life and its relationship with health in heart disease), no differences were found between the three groups; however, the group assigned to the virtual reality format presented significant improvements in its total score between the baseline/initial and final (6 months) moments of the study, and the group assigned to the conventional format presented, only in the first 3 months, significant improvements in the emotional and social dimensions. In the MacNew total score, the group assigned to the virtual reality format, between the baseline/initial and final ( 6 months) moments of the study, according to the mean values, presented a clinically significant variation (a minimum variation of 0.5) [51]. A study conducted by Bocalini et al. [52] showed that there are significant differences between the groups, considering that the group subjected to physical exercise registered higher values in the physical, emotional and social dimensions, in this order, whereas in this study the order was social, physical and emotional. According to the American Heart Association Exercise/American Association of Cardiovascular and Pulmonary Rehabilitation, one of the objectives of the phase II is emotional well-being [53] therefore, the results observed can be due to the fact that the participants, in the phase III, were getting back to normal life after the phase II.

Regarding the DASS 21, despite the fact that there were no significant differences observed between the groups in the results, it should be mentioned that, in the mean values for all the dimensions and total score, there was an improvement from the beginning up to the end ( 6 months) of the study in both IGs, even if it was not significant. According to Whooley et al. [54], the association between depression and cardiovascular disease is complex however, the low level of physical activity is considered a key factor. This justified the importance of evaluating these parameters. According to Bettencourt et al. [55], regardless of the differences in the exercise capacity, patients with coronary artery disease who were integrated in a CR program presented improvements in their anxiety and depression levels. In this way, phase III can act as a tool to preserve these benefits however, the results were not significant in this study.

According to Vieira et al. [22] in this sample, , throughout the study/program, the mean adherence was greater than $65 \%$ in both formats [31], taking into account the three sessions a week, in the first and last 3 months as well as a mean of the 6 months, however, according to Vieira et al. [22], there was a decrease in the last three months. According to Vieira et al. [22], no significant differences were found between the groups, what proves that the adherence rate did not influence the results.

The reduced size of the sample can be referred as a limitation of this study, as well as the difficulty to monitor the adherence to the exercise program objectively. For further studies, we suggest the study of the motivation strategies for the practice of physical exercise, identifying the main triggers for adherence. Age stratification could also be considered, as well as the conducting this type of study with both sexes in the phase II of CR, considering the scholarity level. We also suggest the alliance with other specialized fields for conducting these studies, namely Psychology and the study of other virtual reality instruments. Finally, it would be important in further studies to study the relationship between executive function and quality of life and anxiety, depression and stress, analysing the impact between them.

\section{Conclusions}

In this sample, composed of subjects with coronary artery disease, the prescribed specific exercise program, performed over a sixmonth period in a home context, in the phase III of CR, resulted in improvements in executive function, specifically in the selective attention and conflict resolution ability for the group assigned to the virtual reality format when compared with the CG and conventional format. Therefore, considering the benefits verified, when approaching $C R$, especially when it comprises the use of virtual reality exercise with the Kinect, we believe that the assessment of executive function should be taken into consideration, being the virtual reality a tool to explore in a CR context. This study also reveals the importance of exploring and carrying out the phase III of CR.

\section{Geolocation information}

This study took place in Porto, Portugal, Europe.

\section{References}

[1] Ståhle A, Cider A. Coronary artery disease. In: Börjesson M, Hellenius $M-L$, Jansson $E$, et al., editors. Physical activity in the prevention and treatment of disease. Stockholm: Swedish National Institute of Public Health Distribution; 2010. p. 283-300.

[2] Magalhães S, Viamonte $S$, Ribeiro $M$, et al. Efeitos a longo prazo de um programa de reabilitação cardíaca no controlo dos fatores de risco cardiovasculares [Long-term effects of a cardiac rehabilitation program in the control of cardiovascular risk factors]. Rev Port Cardiol. 2013;32:191-199. Portuguese.

[3] Piepoli MF, Corrà U, Dendale $\mathrm{P}$, et al. Secondary prevention after myocardial infarction: key messages. European Association for Cardiovascular Prevention and Rehabilitation; 2015. Available from: https://www.escardio. org/Sub-specialty-communities/European-Association-forCardiovascular-Prevention-\&-Rehabilitation-(EACPR)/spmi

[4] Antunes HKM, Santos RF, Cassilhas R, et al. Exercício físico e função cognitiva: uma revisão [Physical exercise and cognitive function: a review]. Rev Bras Med Esporte. 2006;12:108-114. Portuguese.

[5] Liu-Ambrose TY, Ashe MC, Graf P, et al. Increased risk of falling in older community-dwelling women with mild cognitive impairment. Phys Ther. 2008;88:1482-1491.

[6] Stanek KM, Gunstad J, Spitznagel MB, et al. Improvements in cognitive function following cardiac rehabilitation for older adults with cardiovascular disease. Int J Neurosci. 2011;121:86-93.

[7] Miyake A, Emerson MJ, Friedman NP. Assessment of executive functions in clinical settings: problems and recommendations. Semin Speech Lang. 2000;21:169-183.

[8] Strauss E, Sherman EMS, Spreen O. A compendium of neuropsychological tests: administration, norms, and commentary. 3rd ed. Oxford: Oxford University Press; 2006. p. 401-405, 477-479, 655-657. 
[9] Banhato EFC, Nascimento ED. Função executiva em idosos: um estudo utilizando subtestes da escala WAIS-III [Executive function in the elderly: a study using WAIS-III scale subtests]. Psico-USF (Impr). 2007;12:65-73. Portuguese.

[10] Chapman SB, Aslan S, Spence JS, et al. Shorter term aerobic exercise improves brain, cognition, and cardiovascular fitness in aging. Front Aging Neurosci. 2013;5:75.

[11] Fechine BRA, Trompieri N. Memória e Envelhecimento: a relação existente entre a memória do idoso e os fatores sócio- demográficos e a prática de atividade física [Memory and aging: the relationship between the elderly's memory and socio-demographic factors and the practice of physical activity]. InterSciencePlace. 2011;1:77-96. Portuguese.

[12] Lange-Asschenfeldta C, Kojdab G. Alzheimer's disease, cerebrovascular dysfunction and the benefits of exercise: from vessels to neurons. Exp Gerontol. 2008;43:499-504.

[13] Fernandes JL, Serrano CV, Jr., Toledo F, et al. Acute and chronic effects of exercise on inflammatory markers and Btype natriuretic peptide in patients with coronary artery disease. Clin Res Cardiol. 2011;100:77-84.

[14] Cuerda RC, Diego IM, Martín JJ, et al. Cardiac rehabilitation programs and health-related quality of life. State of the art. Rev Esp Cardiol. 2012;65:72-79.

[15] Schopfer DW, Forman DE. Benefits of cardiac rehabilitation in older adults. American College of Cardiology; 2016. Available from: http://www.acc.org/latest-in-cardiology/ articles/2016/10/19/09/22/benefits-of-cardiac-rehabilitationin-older-adults

[16] Blair J, Corrigall H, Angus NJ, et al. Home versus hospitalbased cardiac rehabilitation: a systematic review. Rural Remote Health. 2011;11:1532.

[17] Taylor RS, Dalal H, Jolly K, et al. Home-based versus centrebased cardiac rehabilitation. Cochrane Database Syst Rev. 2015;18:CD007130.

[18] Rawstorn JC, Gant N, Direito A, et al. Telehealth exercisebased cardiac rehabilitation: a systematic review and metaanalysis. Heart. 2016;102:1183-1192.

[19] Dahl-Popolizio S, Loman J, Cordes CC. Comparing outcomes of Kinect videogame-based occupational/physical therapy versus usual care. Games Health J. 2014;3:157-161.

[20] Chang $C-Y$, Lange $B$, Zhang $M$, et al. Towards pervasive physical rehabilitation using Microsoft Kinect. Paper presented at: 6th International Conference on Pervasive Computing Technologies for Healthcare (PervasiveHealth) and Workshops; 2012 May 21-24; San Diego, CA.

[21] Vieira Á, Gabriel J, Melo C, et al. Kinect system in homebased cardiovascular rehabilitation. Proc IMechE Part H: J Eng Med. 2017;231:40-47.

[22] Vieira ÁSS, Melo MCDA, Noites ARSSP, et al. The effect of virtual reality on a home-based cardiac rehabilitation program on body composition, lipid profile and eating patterns: a randomized controlled trial. Eur J Integr Med. 2016. [Epub ahead of print]. doi: 10.1016/j.eujim.2016.11.008

[23] Dalal HM, Evans PH, Campbell JL, et al. Home-based versus hospital-based rehabilitation after myocardial infarction: a randomized trial with preference arms-Cornwall Heart Attack Rehabilitation Management Study (CHARMS). Int J Cardiol. 2007;119:202-211.

[24] Dracup K, Evangelista LS, Hamilton MA, et al. Effects of a home-based exercise program on clinical outcomes in heart failure. Am Heart J. 2007;154:877-883.

[25] Jolly K, Tayor RS, Lip GY, et al. Home-based exercise rehabilitation in addition to specialist heart failure nurse care: design, rationale and recruitment to the Birmingham Rehabilitation Uptake Maximisation study for patients with congestive heart failure (BRUM-CHF): a randomised controlled trial. BMC Cardiovasc Disord. 2007;7:9.

[26] Jolly K, Taylor RS, Lip GY, et al. A randomized trial of the addition of home-based exercise to specialist heart failure nurse care: the Birmingham Rehabilitation Uptake Maximisation study for patients with Congestive Heart Failure (BRUM-CHF) study. Eur J Heart Fail. 2009;11: 205-213.

[27] Pescatello LS, Arena R, Riebe D, et al. American College of Sports Medicine's guidelines for exercise testing and prescription. 9th ed. Philadelphia: Wolters Kluwer/Lippincott Williams \& Wilkins; 2014. p. 34-36, 75-85, 241-244.

[28] Vanderlei LC, Pastre CM, Hoshi RA, et al. Basic notions of heart rate variability and its clinical applicability. Rev Bras Cir Cardiovasc. 2009;24:205-217.

[29] Vogels EMHM, Bertram RJJ, Graus JJJ, et al. Clinical practice guidelines for physical therapy in cardiac rehabilitation. KNGF. 2003;8:1-57.

[30] Chen MJ, Fan X, Moe ST. Criterion-related validity of the Borg ratings of perceived exertion scale in healthy individuals: a meta-analysis. J Sports Sci. 2002;20:873-899.

[31] Noites A, Pinto J, Freitas CP, et al. Effects of microcurrents and physical exercise on the abdominal fat in patients with coronary artery disease. Eur J Integr Med. 2015;7:499-507.

[32] Soares JC, Vieira Á, Postolache O, et al. Development of a Kinect rehabilitation system. Int J Online Eng. 2013;9:38-40.

[33] Freitas S, Simões MR, Martins C, et al. Estudos de adaptação do Montreal Cognitive Assessment (MoCA) para a população portuguesa [Studies of adaptation of the Montreal Cognitive Assessment (MoCA) for the Portuguese population]. Aval Psicol. 2010;9:345-357. Portuguese.

[34] Cicchetti DV, Sparrow SA. Developing criteria for establishing interrater reliability of specific items: applications to assessment of adaptive behavior. Am J Ment Defic. 1981;86:127-137.

[35] Arbuthnott K, Frank J. Trail Making Test, part B as a measure of executive control: validation using a set-switching paradigm. J Clin Exp Neuropsychol. 2000;22:518-528.

[36] Tombaugh TN. Trail Making Test A and B: normative data stratified by age and education. Arch Clin Neuropsychol. 2004;19:203-214.

[37] Liu-Ambrose T, Nagamatsu LS, Graf P, et al. Resistance training and executive functions: a 12-month randomized controlled trial. Arch Intern Med. 2010;170:170-178.

[38] Kessels RPC, Van Den Berg E, Ruis C, et al. The backward span of the Corsi Block-Tapping Task and its association with the WAIS-III Digit Span. Assessment. 2008;15:426-434.

[39] Liu-Ambrose T, Ahamed $Y$, Graf $P$, et al. Older fallers with poor working memory overestimate their postural limits. Arch Phys Med Rehabil. 2008;89:1335-1340.

[40] Rand D, Eng JJ, Liu-Ambrose T, et al. Feasibility of a 6month exercise and recreation program to improve executive functioning and memory in individuals with chronic stroke. Neurorehabil Neural Repair. 2010;24:722-729.

[41] Miller EK. The 'working' of working memory. Dialog Clin Neurosci. 2013;15:411-418.

[42] Esgalhado G, Simões F, Pereira H. Versão portuguesa do Teste Stroop de Cores e Palavras: Aferição para a infância e adolescência [Portuguese version of the Stroop Test of colors and words: benchmarking for childhood and adolescence]. Lisboa: Edições Placebo; 2010. 
[43] Esgalhado G, Rocha HP. Efeito do género e da escolaridade no teste stroop: Da infância à adultez jovem [Effect of gender and schooling on the Stroop test: from childhood to young adulthood]. Int J Dev Educ Psychol: INFAD. Rev Psicol. 2012;2:77-86.

[44] Leal ARM. Doenças Cardiovasculares: Prevenção Secundária e Intervenção Comunitária [Cardiovascular diseases: secondary prevention and community intervention] [dissertation]. Porto: Instituto de Ciências Biomédicas Abel Salazar, Universidade do Porto; 2004. p. 64-98.

[45] Tavares N, Madeira R, Henriques A, et al. O efeito de um programa de exercício físico na qualidade de vida em doentes cardíacos [The effect of a physical exercise program on quality of life in cardiac patients]. Rev Portuguesa Saúde Pública. 2013;31:3-10.

[46] Pais-Ribeiro JL, Honrado A, Leal I. Contribuição para o estudo da adaptação portuguesa das escalas de ansiedade, depressão e stress (EADS) de 21 itens de Lovibond e Lovibond [Contribution to the study of Portuguese adaptation of the scales of anxiety, depression and stress (DASS) of 21 items of Lovibond and Lovibond]. Psicol Saúde Doenças. 2004;5:229-239.

[47] Marôco J. Análise estatística com o PASW Statistics (exSPSS) [Statistical analysis with PASW Statistics (ex-SPSS)]. Pêro Pinheiro: ReportNumber, Lda; 2010. p. 113-117, 121-126, 199-210, 213-218, 221-236, 245-252, 321-330, 331-336, 386-418, 432-451.

[48] Kim M, Cho C, Lee C, et al. Effects of cardiac rehabilitation on executive function in sedentary older adults: a systematic review. AJHS. 2016;11:98-110.

[49] Pereira PPS, Voos MC, Machado MAS, et al. Interferência mútua entre atividade visual e atividade motora em jovens

e idosos [Mutual interference between visual activity and motor activity in young and old]. Fisioter Pesqui. 2008;15:142-148.

[50] Kramer AF, Colcombe SJ, McAuley E, et al. Fitness, aging and neurocognitive function. Neurobiol Aging. 2005; 26:124-127.

[51] Dixon T, Lim LL, Oldridge NB. The MacNew heart disease health-related quality of life instrument: reference data for users. Qual Life Res. 2002;11:173-183.

[52] Bocalini DS, Santos L, Serra AJ. Physical exercise improves the functional capacity and quality of life in patients with heart failure. Clinics (Sao Paulo). 2008;63:437-442.

[53] Balady GJ, Williams MA, Ades PA, et al. Core Components of Cardiac Rehabilitation/Secondary Prevention Programs: 2007 Update: a scientific statement from the American Heart Association Exercise, Cardiac Rehabilitation, and Prevention Committee, the Council on Clinical Cardiology; the Councils on Cardiovascular Nursing, Epidemiology and Prevention, and Nutrition, Physical Activity, and Metabolism; and the American Association of Cardiovascular and Pulmonary Rehabilitation. Circulation. 2007;115:2675-2682.

[54] Whooley MA, de Jonge P, Vittinghoff $E$, et al. Depressive symptoms, health behaviors, and risk of cardiovascular events in patients with coronary heart disease. JAMA. 2008;300:2379-2388.

[55] Bettencourt N, Dias C, Sampaio PM, et al. Impacto da Reabilitação Cardíaca na Qualidade-de-Vida e Sintomatologia Depressiva após Síndroma Coronária Aguda [Impact of cardiac rehabilitation on quality-of-life and depressive symptoms after acute coronary syndrome]. Rev Port Cardiol. 2005;24:687-696. 